\title{
Symmetry background of the fractional Aharonov-Bohm oscillation and an oscillation in dipole-transitions of narrow quantum rings with a few-electrons
}

\author{
C. G. Bao*, G. M. Huang, and Y. M. Liu \\ The State Key Laboratory of Optoelectronic Materials and Technologies, \\ and Department of Physics, Zhongshan University, Guangzhou, 510275, P.R. China
}

\begin{abstract}
The low-lying spectrum of a 3-electron narrow ring has been analyzed analytically. A phasediagram for the ground state band against the magnetic field and the radius of the ring is obtained. The symmetry background of the fractional Aharonov-Bohm oscillation has been revealed. A very strong oscillation in the dipole transition is found. The discussion can be generalized to N-electron rings.

PACS numbers: 73.23.Ra, 78.66. -w

*Corresponding Author.
\end{abstract}

Quantum rings with a radius from 20 to $120 \mathrm{~nm}$ containing only a few electrons can now be fabricated in laboratories $^{1,2}$. When a magnetic field $B$ is applied, interesting physical phenomena, e.g., persistent currents and Aharonov-Bohm oscillation (ABO) with a period $\Phi_{0}=h c / e$, the flux quanta, have been observed ${ }^{3,4}$. In particular, the fractional ABO (FABO) with a period a fraction of $\Phi_{0}$ has been observed recently ${ }^{2,5}$. The experimental observations, including the fractional periodicity, can in general be explained theoretically. Energy spectra and electronic correlations have been studied in quite detail ${ }^{6-11}$. Nonetheless, in the previous study the emphasis was played in the dynamical aspect, the effect of symmetry was less touched. However, this effect is believed to be in general important to few-body systems $^{12-16}$.

To remedy, the motivation of this paper is to clarify how the internal structures and physical properties are affected by symmetry. For this purpose, we shall analyze the nodal structures of wave functions, thereby to clarify the symmetry background of the FABO and to clarify the regularity appearing in the spectra. Furthermore, since the energy levels are affected by symmetry, the optical properties might be affected as well, this is also a point to be studied. In this paper only narrow rings are concerned, they are treated as one-dimensional.

Let a magnetic field $B$ be perpendicular to the plane of a ring containing $N$ electrons, let $\theta_{j}$ be the azimuthal angle of the $j-t h$ electron, let a set of relative angles be defined as $\varphi_{1}=\theta_{2}-\theta_{1}, \varphi_{2}=\theta_{3}-\left(\theta_{2}+\theta_{1}\right) / 2, \cdots \cdots$, $\varphi_{N-1}=\theta_{N}-\sum_{i=1}^{N-1} \theta_{i} /(N-1)$, and $\theta_{C}=\sum_{i=1}^{N} \theta_{i} / N$. The Hamiltonian reads

$$
H=H_{\text {coll }}+H_{\text {in }}+H_{Z e e m a n}
$$

where

$$
H_{\text {coll }}=D\left(-i \frac{\partial}{\partial \theta_{C}}+\alpha B\right)^{2}
$$

is for the collective motion, $D=\frac{\hbar^{2}}{2 N m^{*} R^{2}}, \quad \alpha=$ $\frac{N e R^{2}}{2 \hbar C}=\frac{N \sigma}{\Phi_{0}}, m^{*}$ is the effective mass, $\sigma$ the area of the ring.

$H_{i n}=\sum_{j}^{N-1} \frac{\hbar^{2}}{2 \mu_{j} R^{2}}\left(-i \frac{\partial}{\partial \varphi_{j}}\right)^{2}+\sum_{j<i} V_{j i}$

is the internal Hamiltonian not depending on $B, \mu_{j}=$ $j m^{*} /(j+1)$ is the reduced mass, and the interaction is adjusted as

$$
V_{j i}=\frac{e^{2}}{2 \varepsilon \sqrt{d^{2}+R^{2} \sin ^{2}\left(\left(\theta_{i}-\theta_{j}\right) / 2\right)}}
$$

where $\varepsilon$ is the dielectric constant and the parameter $d$ is introduced to account for the effect of finite thickness of the ring ${ }^{7}$. The Zeeman energy is $H_{Z \text { eeman }}=\gamma B \cdot S_{Z}$, where $\gamma=g \mu_{B}$, and $S_{Z}$ is the Z-component of the total spin $S$.

It is clear from (2) that the collective motion is equivalent to the motion of a single particle with a mass $\mathrm{Nm}^{*}$ and a charge $\mathrm{Ne}$. However, $H_{i n}$ remains to be diagonalized. Let the eigenstates of $H_{i n}$ be called internal states, the permutation symmetries of their spatial parts depend on $S$. Thus, it is sure that the internal states depend on $S$. On the other hand, the orbital angular momentum $L$ is an eigenvalue of $H_{\text {coll }}$, it describes the collective motion, therefore the internal states might not depend on $L$. However, as we shall see, they depend on $L$ in a very special way. In what follows $L$ is considered as positive, and $B$ is negative.

We shall study mainly the case $N=3$, the basis functions (not yet normalized)

$$
e^{i\left(k_{1} \theta_{1}+k_{2} \theta_{2}+k_{3} \theta_{3}\right)}=e^{i L \theta_{C}} \cdot e^{i\left(k_{a} \varphi_{1}+k_{b} \varphi_{2}\right)}
$$

can be adopted, where $L=k_{1}+k_{2}+k_{3}, k_{a}=\left(k_{2}-\right.$ $\left.k_{1}\right) / 2$, and $k_{b}=k_{3}-L / 3$. Since $k_{1}, k_{2}$, and $k_{3}$ must be integer to assure the periodicity, $k_{b}$ would be an integer if $L=3 J$ (where $J$ is an arbitrary integer) and therefore the period of $\varphi_{2}$ is $2 \pi$. Whereas, if $L \neq 3 J, k_{b}$ would not be an integer and the period of $\varphi_{2}$ is $6 \pi$. Obviously, the two kinds of periodicity of $\varphi_{2}$ lead to two kinds of internal structures. Thus, together with the two choices $S=1 / 2$ and $3 / 2$, the internal states can be classified into four internal series $\psi_{Q S, i}$, where $Q=+1$ implies the case $L=3 J, Q=-1$ implies $L \neq 3 J$. In other words, $Q$ denotes a specific subset of $L$, the internal states do not depend on the value of $L$ but on $Q$.

Due to eq.(1), once $B$ and the energy $E_{Q S, i}$ of an internal state $\psi_{Q S, i}$ is known, a series of energy levels with angular momenta $L$ belonging to the subset $Q$ can be generated as 


$$
E=D(L+\alpha B)^{2}+E_{Q S, i}+\gamma B \cdot S_{Z}
$$

each is associated with the eigenstate

$$
\Psi=\frac{1}{\sqrt{2 \pi}} e^{i L \theta_{C}} \psi_{Q S, i}
$$

It is noted that $H_{\text {in }}$ does not depend on $B$, therefore $E_{Q S, i}$ does not depend on $B$ also.

Let us study the 3-electron ring numerically as an example. Let $m^{*}=0.063 m_{e}, \varepsilon=12.4$ (for InGaAs), and $d=0.05 R$ (the qualitative results are not sensitive to $d)$. For the diagonalization of $H$, the basis functions $e^{i\left(k_{1} \theta_{1}+k_{2} \theta_{2}+k_{3} \theta_{3}\right)} /(2 \pi)^{3 / 2}$ are used, where $k_{i}$ are integers, each is ranged from -20 to 20 . This is sufficient to obtain accurate solutions. After the diagonalization, the eigenenergies $E$ are obtained. From $E$ and from eq.(6) $E_{Q S, i}$ can be extracted and four internal series are found just as predicted, they are listed in Table 1 .

Table 1, Internal energies $E_{Q S, i}$ of a 3-electron ring (in $\mathrm{meV}$ ), the cases with $i=1$ and 2 are listed.

\begin{tabular}{|c|c|c|c|}
\hline$(\mathrm{Q}, \mathrm{S})$ & $R=30 n m$ & $R=90 n m$ \\
\hline$\left(1, \frac{3}{2}\right)$ & $9.1442, \quad 15.1320, \cdots$ & 2.6533, & $3.6085, \cdots$ \\
\hline$\left(-1, \frac{3}{2}\right)$ & $11.9455, \quad 15.4173, \cdots$ & $3.1107, \quad 3.6336, \cdots$ \\
\hline$\left(1, \frac{1}{2}\right)$ & $11.8278, \quad 11.8827, \cdots$ & $3.1101, \quad 3.1105, \cdots$ \\
\hline$\left(-1, \frac{1}{2}\right)$ & $9.1161, \quad 11.8567, \cdots$ & $2.6532, \quad 3.1103, \cdots$ \\
\hline
\end{tabular}

It is clear from this table that, not matter how $R$ is, $E_{1,3 / 2,1}$ and $E_{-1,1 / 2,1}$ are particularly low. Using only these two and using eq.(6) the low-lying spectrum against $B$ can be exactly and entirely generated as shown in Fig.1. It is surprising that such a complicated spectrum contains only two internal states. Let $L_{o}$ and $S_{o}$ denote the quantum numbers of the ground state. In Fig.1 the ground state band (GSB) contains many segments, each has its own $L_{o}$ and $S_{o}$. When $S_{o}=3 / 2$, $L_{0}=3 J$; when $S_{o}=1 / 2, L_{0} \neq 3 J$. When $|B|$ increases, $L_{o}$ will increase and the ground state will transit from one segment to its neighboring segment step by step.

Let $\chi_{\uparrow}(i)$ and $\chi_{\downarrow}(i)$ denote the up and down spinstates. Let $S_{Z}=1 / 2$ and $\chi_{\uparrow \uparrow \downarrow}(123) \equiv \chi_{\uparrow}(1) \chi_{\uparrow}(2) \chi_{\downarrow}(3)$, then the antisymmetrized internal states can be expanded as ${ }^{16}$

$\psi_{Q S, i} \equiv \psi_{\uparrow \uparrow \downarrow}(123) \chi_{\uparrow \uparrow \downarrow}(123)+\psi_{\uparrow \uparrow \downarrow}(231) \chi_{\uparrow \uparrow \downarrow}(231)+$ $\psi_{\uparrow \uparrow \downarrow}(312) \chi_{\uparrow \uparrow \downarrow}(312)$

Since the three terms in (8) are different only in the names of particle, the observation of one of them is sufficient. The norm $\left|\psi_{\uparrow \uparrow \downarrow}(123)\right|$ of $\psi_{Q S, i}$ with $i=1$ and 2 are plotted in Fig.2 as a function of $\varphi_{2}$. In this choice, we are observing the motion of the spin-down electron relative to the two spin-up electrons. It is reminded that, if a pair of electrons are close to each other, the energy would increase due to the $e-e$ repulsion. Thus, the best configuration is the equilateral triangle $(E T)$ which is associated with the minimum of the total interaction energy. From the point of dynamics, all the first-states ( $i=1$ ) would pursue the $E T$ so as to lower their energies. However, only the two series with $(Q S)=\left(1, \frac{3}{2}\right)$ and $\left(-1, \frac{1}{2}\right)$ succeed, they have a peak at $\varphi_{2}=\pi$ which

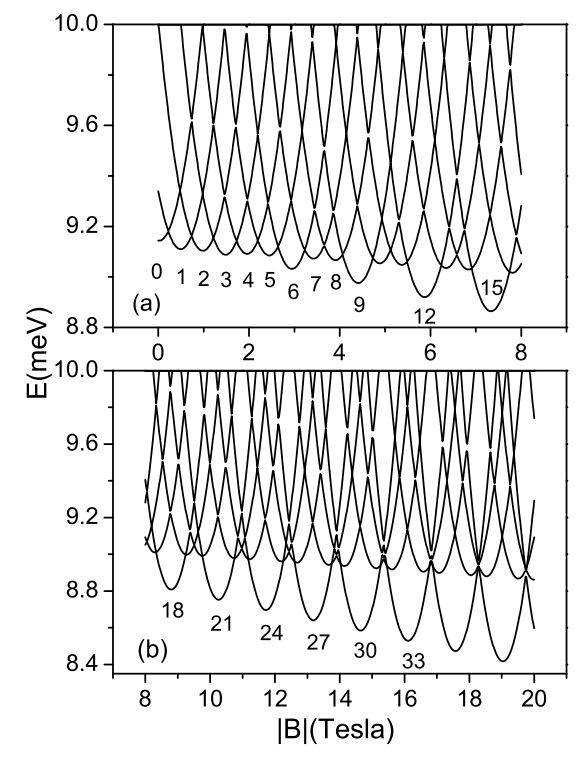

FIG. 1: Evolution of low-lying levels of a 3-electron ring with $R=30 \mathrm{~nm}$ against $B$. The orbital angular momentum of the ground state $L_{o}$ are marked by the levels.

corresponds to an ET (Fig.2a and 2d). Whereas there is a node at the $E T$ in the other two series . Evidently, the node appearing at the minimum of potential energy would push the wave function away from the minimum and thereby cause the excitation of an oscillation back and forth around the minimum as an equilibrium point, this would cause a great increase in energy. This explains why the other two series are remarkably higher.

The appearance of the node has a profound background of symmetry. In general, if the $N$ electrons form a $\mathrm{N}$-sided equilateral polygon $(E P)$ in the ring, the total interaction energy would be minimized. However, at this configuration, a rotation by $2 \pi / N$ is equivalent to a cyclic permutation of coordinates. It was proved in ref.[15] that this equivalence leads to a constraint. As a result, the wave functions of $S=N / 2$ states would have an inherent node at the $E P$ if $L \neq N G$, where $G$ is an integer (half-integer) if $N$ is odd (even). Alternatively, when $S=N / 2-1$, the node would appear if $L=N G$, . This is called an EP-prohibition (PEP). Obviously, if the set of orbital angular momenta contained in an internal series are free from the PEP, the series is allowed to pursue the favourable EP geometry, therefore its first states $(i=1)$ would be particularly low. This is the symmetry background that, when $N=3$, the $(Q, S)=(1,3 / 2)$ and $(-1,1 / 2)$ first-states are low (cf. Table 1$)$.

Based on eq.(6), the feature of the spectra can be explained analytically. When $|B|$ is small, Fig.1 shows that, in the GSB, the difference in $L_{o}$ of two neighboring segments is one, i.e., $L_{o}$ is compactly aligned. Each segment is ranged from $B_{a}\left(L_{o}\right)$ to $B_{b}\left(L_{o}\right)$ and contains a minimum of energy at $B_{\min }\left(L_{o}\right) . B_{a}$ and $B_{b}$ can be de- 
rived from eq.(6) together with the condition that neighboring segments have the same energy at the boundaries. Besides, $B_{\min }$ can also be derived from eq.(6) and from $d E / d B=0$. Due to $\gamma$ being small, we have

$$
B_{\min }\left(L_{o}\right)=-\left(2 D \alpha L_{o}+\gamma S_{o}\right) / 2 D \alpha^{2} \approx-L_{o} / \alpha,
$$

Accordingly, the distance between the minima of two neighboring segments is $B_{\min }\left(L_{o}\right)-B_{\min }\left(L_{o}+1\right)$ $\approx 1 / \alpha=\Phi_{o} / 3 \sigma$, which is just the period of the $\mathrm{FABO}^{2,5}$. Thus, the compact alignment leads to the FABO.

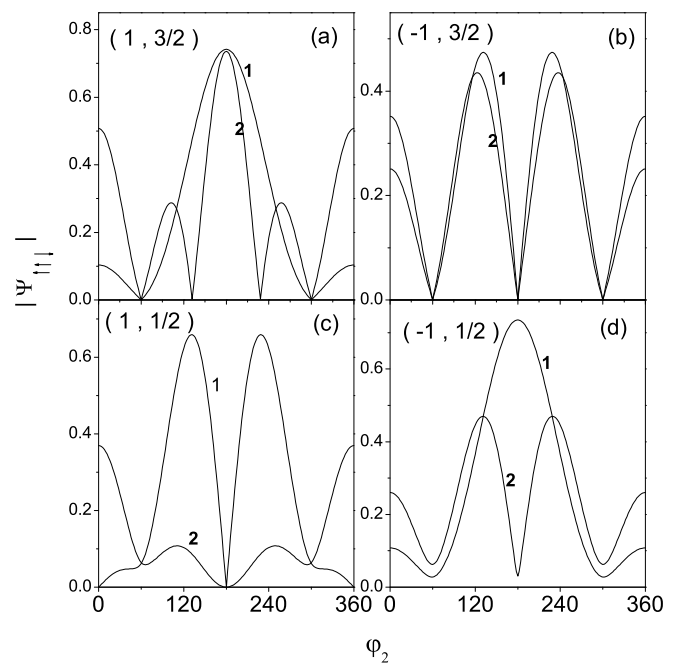

FIG. 2: $\left|\psi_{\uparrow \uparrow \downarrow}\right|$ component of the internal states $\psi_{Q S, i}$ plotted against $\varphi_{2}, \varphi_{1}$ is fixed at $120^{\circ}, R=30 \mathrm{~nm}$, the label $(Q S)$ are marked in the upper corner and $i$ by the curves.

Fig.1 shows that, due to the Zeeman effect, the increase of $|B|$ would enlarge the segments with $S=3 / 2$ (i.e., $L_{o}=3 J$ ), while those with $S=1 / 2$ (i.e., $L_{o} \neq 3 J$ ) would become smaller and smaller. When $|B|$ is sufficiently large, the minima of the $S=1 / 2$ segments would disappear. This would begin to occur if $B_{b}(3 J)=B_{\min }(3 J+1)$. In this case we define a critical value $B_{\text {crit } 1}=\left|B_{a}(3 J)\right|$. When $|B|$ is even larger, all the $S=1 / 2$ segments would disappear. This would begin to occur if $B_{b}\left(3 J^{\prime}\right)=B_{a}\left(3 J^{\prime}+3\right)$. In this case we define another critical value $B_{\text {crit } 2}=\left|B_{a}\left(3 J^{\prime}\right)\right|$. E.g., for Fig. $1, B_{\text {crit } 1}=9.8$ and $B_{\text {crit } 2}=18.2$. Evidently, when $|B|>B_{\text {crit } 1}$, due to the miss of the minima of the $S=1 / 2$ segments, the distance between two adjacent minima (evaluated from (9)) is changed from $\Phi_{o} / 3 \sigma$ to $\Phi_{o} / \sigma$. Thus, the period of the normal ABO recovers, and $B_{c r i t}$ is the boundary separating the fractional and normal ABO. When $|B|>B_{\text {crit } 2}$, all the $S=1 / 2$ segments disappear, and the GSB becomes purely polarized. Thus $B_{\text {crit } 2}$ is the boundary separating the partial and full polarization.

Based on the analytical expression eq.(6), the values of $B_{\text {crit } 1}$ and $B_{\text {crit } 2}$ can be obtained analytically, they depend on the radius $R$. . The numerical results are plotted in Fig.3, which is a phase diagram containing three regions. The FABO appears only in region (I), thus it

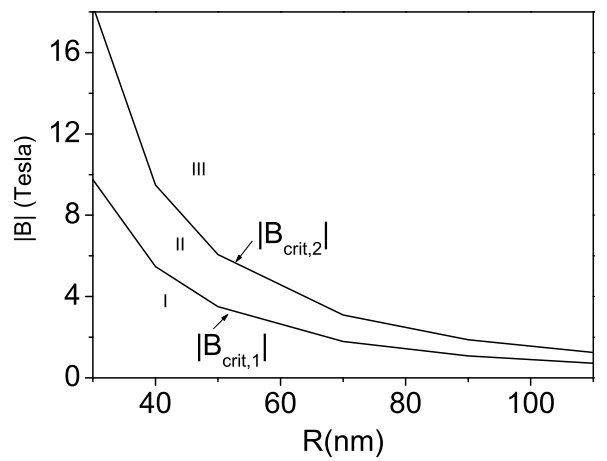

FIG. 3: The evolution of $B_{\text {crit } 1}$ and $B_{\text {crit } 2}$ against $R$.

emerges only when $|B|$ is weak. However, for the rings with a small $R$ (say, $R<30 \mathrm{~nm}$ ), the FABO exists even if $B$ is strong.

Incidentally, let the orbital angular momenta of the GSB associated with $B_{\text {crit } 1}$ and $B_{\text {crit } 2}$ be denoted as $L_{c r i t, 1}$ and $L_{c r i t, 2}$. It was found that both $L_{c r i t, 1}$ and $L_{\text {crit }, 2}$ depend on $R$ very weakly. When $R$ is ranged from 30 and $120, L_{c r i t, 1}$ and $L_{c r i t, 2}$ remain to be 21 and 36 , respectively.

The transitions of $L_{o}$ and $S_{o}$ in the GSB would lead not only to the oscillation of ground state energy, but also an oscillation in dipole radiation. Let the temperature $T$ be very low (e.g., $T \leq 0.01 K$ ) so that the system is mainly in its ground state. When dipole transitions between the ground state and a final state occurs both $S_{o}$ and its Z-component are conserved, and the difference in angular momenta $L_{f}-L_{o}$ is \pm 1 , ( the subscript $f$ denotes the final state). The difference in energies, from eq.(6), is

$E_{f}-E_{o}=E_{Q_{f}, S_{o}, i}-E_{Q_{o} S_{o}, 1}+D\left(1 \pm 2\left(L_{o}+\alpha B\right)\right)$

Let us focus on the most infrared radiation, i.e., the low energy limit denoted as $\lim \left(E_{f}-E_{o}\right)$. When $L_{o}=3 J$, we have $\left(Q_{o}, S_{o}\right)=(1,3 / 2)$. Since meanwhile $L_{f}=3 J \pm$ 1 , thus $Q_{f}=-1$. Since $E_{-1,3 / 2,1}$ is associated with the internal series suffering the PEP, it is high and therefore leads to a big $\lim \left(E_{f}-E_{o}\right)$ (cf. Table 1). When $L_{o}=$ $3 J-1$, we have $\left(Q_{o}, S_{o}\right)=(-1,1 / 2)$. Meanwhile $L_{f}$ has two choices, $3 J-2$ or $3 J$. The former choice has $Q_{f}=$ -1 , thus the ground state and the final state would have the same internal state $\psi_{-1, \frac{1}{2}, 1}$ with the same internal energy $E_{-1,1 / 2,1}$, and therefore leads to a small $\lim \left(E_{f}-\right.$ $\left.E_{o}\right)$. The latter choice has $Q_{f}=+1$. However, $E_{1,1 / 2,1}$ is also pushed up by the PEP, therefore this choice leads to a large $E_{f}-E_{o}$ and can be neglected. Similarly, When $L_{o}=3 J+1$, a small $\lim \left(E_{f}-E_{o}\right)$ exists. The low energy limit of the radiation absorbed by the GSB is plotted in Fig.4. During the increase of $|B|<B_{\text {crit1 } 1}$, each time when $B$ lies in a segment with $L_{o}=3 J$, the $\operatorname{limit} \lim \left(E_{f}-E_{o}\right)$ jumps up remarkably and then jumps down afterward. This causes a very strong oscillation much stronger than the oscillation of the ground state energy. This oscillation would become even stronger 
when $R$ becomes smaller. The period of the oscillation is simply $B_{\min }\left(L_{o}\right)-B_{\min }\left(L_{o}+3\right) \approx \Phi_{o} / \sigma$, the same as the normal ABO. However, when $|B|>B_{\text {crit } 1}$, the GSB is dominated by $L_{o}=3 J$ states, and the oscillation would disappear because the $\lim \left(E_{f}-E_{o}\right)$ do not jump down again.

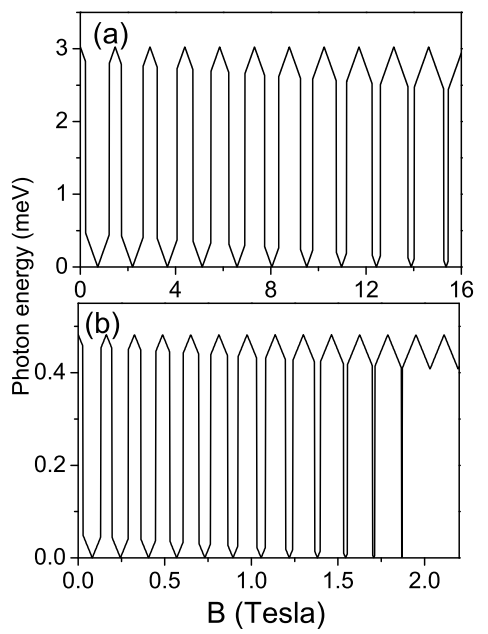

FIG. 4: Oscillation of the low energy limit of the radiation of the ground state against $B . \quad R=30 \mathrm{~nm}$ (a) and $90 \mathrm{~nm}$ (b).

In summary, the narrow rings with three electrons have been studied. The following findings are noticeable.

(1) When the relative angles $\varphi_{i}$ are introduced to describe the internal states, the periods of $\varphi_{i}$ depend on $L$. Therefore, the internal states depend on $L$ in a specific way, and can be classified into a few series based on the periodicity. A specific subset of $L$ is allowed in a series.

(2) The structures of distinct series of internal states are affected by symmetry in different ways. In particular, two favourable series are free from the PEP, their first-states are particularly low. These two firststates are sufficient to generate exactly the complete lowlying spectra. This is an interesting point, thereby the spectra can be understood analytically.

(3) The $L$ allowed by the two first-states are complementary, they cover the whole range of $L$. When $|B|$ increases but $\leq B_{\text {crit } 1}$, the two first-states appear in the GSB alternatively. As a result, $L_{o}$ increases one-byone, i.e., they are compactly aligned. This leads to the FABO. However, when $B>B_{\text {crit } 1}$, the internal state with $S=1 / 2$ disappears from the GSB. As a result, $L_{o}$ increases each time by three, thus the normal $\mathrm{ABO}$ recovers.

(4) When $|B|<B_{\text {crit } 1}$, a strong oscillation of the low energy limit of the radiation of the GSB was found. The amplitude of this oscillation is much stronger than that of the ground state energy. This is also a very interesting phenomenon.

Although the above findings are extracted from 3electron rings, they hold also for $N>3$ rings. It implies that the internal states can also be classified according to the periods of $\varphi_{i}$, thus they depend on $L$ in a special way.
Some internal series are free from the PEP, and the lowlying spectra are generated by the first-states of them. When $|B|$ is small, $L_{o}$ is compactly aligned, the FABO would emerge. When $|B|$ is large $\left(|B|>B_{\text {crit } 2}\right)$, the GSB is fully polarized and $L_{o}$ increases each time by $N$, thus the normal $\mathrm{ABO}$ recovers. Before the full polarization , there would be a domain of $B$ in which the GSB is dominated by both $S=N / 2$ and $N / 2-1$ segments. When $B$ is given in one of the $S=N / 2$ segment, the most infrared radiation of the ground state is associated with a transition of the internal state from a PEP-free series to a series suffering the PEP, and thus results in a large $\lim \left(E_{f}-E_{o}\right)$, while in the neighboring segments the $\lim \left(E_{f}-E_{o}\right)$ is small. This leads to the occurrence of the above mentioned optical oscillation. Incidentally, this oscillation does not appear when $N=1$ or 2 .

Nonetheless, the cases with $N>3$ and the cases of broad rings have to be further studied.

Acknowledgment, This work is supported by the NSFC of China under the grants 90306016 and 10174098.

1, A. Lorke, R.J. Luyken, A.O. Govorov, J.P. Kotthaus, J.M. Garcia, and P.M. Petroff, Phys. Rev. Lett. 84, 2223 (2000).

2, U.F. Keyser, C. Fühner, S. Borck, R.J. Haug, M. Bichler, G. Abstreiter, and W. Wegscheider, Phys. Rev. Lett. 90, 196601 (2003)

3, D. Mailly, C. Chapelier, and A. Benoit, Phys. Rev. Lett. 70, 2020 (1993)

4, A. Fuhrer, S. Lüscher, T. Ihn, T. Heinzel, K. Ensslin, W. Wegscheider, and M. Bichler, Nature (London) 413, $822(2001)$

5, S.Viefers, P. Koskinen, P. Singha Deo, M. Manninen, Physica E 21, 1 (2004)

6, K. Niemelä, P. Pietiläinen, P. Hyvönen, and T. Chakraborty, Europhys. Lett. 36, 533 (1996)

7, M. Korkusinski, P. Hawrylak, and M. Bayer, Phys. Stat. Sol. B 234, 273 (2002)

8, Z. Barticevic, G. Fuster, and M. Pacheco, Phys. Rev. B 65, 193307 (2002)

9, M. Ferconi and G.Vignale, Phys. Rev. B 50, 14722 (1994).

10, Li. Serra, M. Barranco, A. Emperador, M. Pi, and E. Lipparini, Phys. Rev. B 59, 15290 (1999)

11, A. Emperador, F. Pederiva, and E. Lipparini, Phys. Rev. B 68, 115312 (2003)

12, C.G. Bao, Few-Body Systems, 13, 41 (1992)

13, C.G. Bao, W.F. Xie, C.D. Lin, J. Phys. B: At. Mol. Opt. Phys. 27, L193 (1994)

14, W.Y. Ruan, Y.Y. Liu, C.G. Bao, Z.Q. Zhang, Phys. Rev. B 51, 4792 (1995)

15, C.G. Bao, Phys. Rev. Lett. 79, 3475 (1997)

16, C.G. Bao, Z.Phys. D 22, 557 (1992)

17, H. Weyl, The Classical Groups (Princeton University Press, Princeton, N.J., 1946)

18, D.E. Rutherford, Substitutional Analysis (Edinburgh University Press, Edinburgh, 1948) 
